Revista Signos

2010 / 43

Número Especial

Monográfico $\mathrm{N}^{\circ} 2$

279-294

\title{
De los actos comunicativos de poder a los actos comunicativos dialógicos en las aulas organizadas en grupos interactivos
}

\author{
Esther Oliver \\ Universidad de Barcelona \\ España
}

\author{
Suzanne Gatt \\ Universidad de Malta \\ Malta
}

Resumen: Los grupos interactivos son una de las formas de organización de las aulas que está obteniendo más éxito en Europa en la superación del fracaso escolar y los problemas de convivencia. Este artículo muestra cómo en el análisis llevado a cabo en INCLUD-ED se ha encontrado una de las claves para ese éxito: la continua sustitución de actos comunicativos de poder, típicos de las aulas tradicionales, por actos comunicativos dialógicos. Los actos comunicativos de poder que afectan al alumnado excluido en las aulas ordinarias, en espacios de educación compensatoria, o apartado de los espacios regulares a través de otras formas de segregación educativa, son transformados en actos comunicativos dialógicos basados en el diálogo igualitario (y que incluyen las condiciones de sinceridad y consenso). Por un lado, el artículo analiza en profundidad actuaciones educativas relacionadas con el fracaso escolar y basadas en actos comunicativos de poder, donde predominan las interacciones de poder. Se ha prestado especial atención a la presencia de actos comunicativos de poder en dinámicas de segregación que afectan principalmente a estudiantes de grupos vulnerables. Por otro lado, se estudian los grupos interactivos como una actuación educativa de éxito donde predominan las interacciones dialógicas basadas en un diálogo igualitario, y donde se consigue un mayor impacto en la mejora de los resultados educativos. Como resultado de nuestro análisis, se identifican los principales elementos que explican la relación tanto entre los actos comunicativos de poder y el fracaso escolar como entre los actos comunicativos dialógicos y el éxito educativo.

Palabras Clave: Interacciones dialógicas, interacciones de poder, diálogo igualitario, grupos interactivos, actuaciones educativas de éxito.

Recibido:

20-IV-2010

Aceptado:

9-VII-2010
Correspondencia: Esther Oliver (estheroliver@ub.edu). Departamento de Teoría Sociológica, Filosofía del Derecho y Metodología de las Ciencias Sociales. Facultad de Economía y Empresa, Universidad de Barcelona. Diagonal Nord, Diagonal 690, 08034 Barcelona, España. 


\title{
From power-related communicative acts to dialogic communicative acts in classrooms organised in interactive groups
}

\begin{abstract}
Interactive groups is the form of classroom organisation that is having quite a success in Europe in terms of overcoming school failure and coexistence problems. The analysis carried out in the INCLUD-ED project identified that one of the keys to this success was the continuous replacement of power-related communicative acts, which are typical of traditional classrooms, with dialogic communicative acts. The power-related communicative acts, which affect students either excluded from ordinary classrooms or placed in compensatory education classes or removed from mainstream areas through other forms of educational segregation, are transformed into dialogic communicative acts in groups based on egalitarian dialogue (which involves the conditions of sincerity and consensus). This article develops the provious argument through an in-depth analysis of educational actions linked to school failure and based on power-related communicative acts, in which there is a predominance of power-related interaction. Special attention was paid to power-related communicative acts in segregationist practices that mainly affect students belonging to vulnerable groups. Furthermore, interactive groups are studied as a successful educational action in which dialogic interaction predominates, based on egalitarian dialogue. Consequently, a greater impact on an improvement in educational results can be achieved using this form of organisation. As a result of our analysis, the main elements which explain the link both between power-related communicative acts and school failure, and between dialogic communicative acts and educational success are identified.
\end{abstract}

Key Words: Dialogic interactions, power interactions, egalitarian dialogue, interactive groups, successful educational actions.

\section{INTRODUCCIÓN}

En un aula una profesora está realizando una clase de inglés. Ahmed, un alumno de Marruecos que hace unas semanas que ha llegado, está en la clase. La profesora le da un dossier de castellano para que, mientras los demás trabajan inglés, él refuerce la lengua castellana. AAhmed no se le ha explicado el por qué, solo se le ha dicho que él no puede hacer inglés porque primero tiene que aprender bien el castellano. La familia no está informada. El niño no aprende ni inglés ni castellano, porque la profesora, aunque lo intenta, no puede dedicarle tiempo para que consiga realizar las actividades del dossier de forma comprensiva.

Esta es una de las actuaciones en las que se dan actos comunicativos de poder, relatada por una de las personas colaboradoras del INCLUD-ED consultadas para la realización de este artículo. Como este, se pueden dar diariamente multitud de actos comunicativos de poder en las escuelas, que contribuyen a explicar el fracaso escolar.

Nuestra aportación se sitúa en el ámbito de la discusión entre actos de habla y actos comunicativos que se ha dado entre teóricos como Austin (1971), Searle (2001) y Habermas (1984, 
1987). Partimos de la aportación que Soler y CREA (Aubert, Flecha, García, Flecha \& Racionero, 2008) realizan a esta discusión con la diferenciación entre actos comunicativos de poder y actos comunicativos dialógicos. Vamos a profundizar en esta diferenciación para obtener nuevos elementos que ayuden a identificar componentes de las actuaciones educativas que conducen al fracaso educativo. Al mismo tiempo, vamos a analizar los actos comunicativos presentes en las actuaciones educativas inclusoras que están generando éxito escolar para todos y todas las niñas. Asimismo, analizaremos la presencia de interacciones dialógicas e interacciones de poder tanto en los actos comunicativos de poder como en los dialógicos, evidenciando la influencia del contexto y la estructura social en la articulación de estos diálogos e interacciones entre las personas. Al mismo tiempo, observaremos también el papel que los agentes sociales, en este caso, los niños y niñas y las personas adultas que interaccionan con ellos y ellas, tienen en el cuestionamiento de esta estructura y en la articulación de nuevas actuaciones lingüísticas que permiten crear realidades más dialógicas en las escuelas (Flecha, 2009; Gatt, Ojala \& Soler, en proceso).

En el caso que acabamos de ilustrar, se produce un acto comunicativo de poder porque ni a Ahmed ni a su familia se les ha explicado los motivos por los que no se le permite al chico participar de la clase de inglés como al resto de sus compañeros y compañeras. Entre estos motivos puede haber la creencia por parte del profesorado de que Ahmed no está preparado para seguir el mismo ritmo que el resto de alumnado, o bien, pueden existir estereotipos o prejuicios en la escuela en relación a las capacidades de la población de origen inmigrante. Este caso puede ser también un ejemplo del lenguaje de serpiente (Aubert, García \& Racionero, 2009) presente en nuestras escuelas, que se manifiesta cuando no perseguimos conseguir para los demás niños y niñas lo que querríamos para nuestros hijos e hijas.

Detrás de la decisión de no incluir por el momento a Ahmed en el aprendizaje del inglés, no hay evidencias científicas que demuestren que con esa estrategia acabará dominando las dos lenguas; por el contrario sí que puede haber bajas expectativas del profesorado que se transmiten en la comunicación acerca de sus posibilidades de aprendizaje. En este caso, la falta de sinceridad sobre las razones de apartar a Ahmed de la actividad del grupo clase y la falta de consenso con la familia sobre los argumentos que justifican esta práctica pueden contribuir a explicar porqué el chico no aprende ni castellano ni inglés.

Concretamente, de entre los resultados obtenidos en INCLUD-ED y del testimonio de cuatro profesionales de la educación en el ámbito de la educación primaria y secundaria que han colaborado en el proyecto de investigación, en este artículo recuperamos aquellas evidencias que nos ayudan a identificar los elementos de los actos comunicativos que inciden en este fracaso o bien que contribuyen a la mejora de los resultados académicos. 
Los resultados que presentamos muestran la existencia de un lenguaje positivo y la promoción de las interacciones dialógicas en determinadas formas de participación de familiares y voluntariado en las aulas y otros espacios de la escuela. Estos diálogos tienen un impacto positivo en los logros académicos y la convivencia (Flecha, García, Gómez \& Latorre, 2009).

La actuación de éxito que se analiza con mayor profundidad en este artículo son los grupos interactivos (Racionero \& Valls, 2007). Esta forma de agrupación inclusiva del alumnado consiste en la distribución de los alumnos y alumnas en pequeños grupos heterogéneos dentro de la misma aula, junto con una reorganización de los recursos humanos existentes en la escuela. La dotación de diferentes personas adultas incluyendo la entrada y participación plena de familiares y personas voluntarias no se utiliza para separar al alumnado en función de su nivel o competencia ni para proveer adaptaciones curriculares a los diferentes alumnos y alumnas, sino para promover interacciones de apoyo y aprendizaje mutuo entre el alumnado en actividades comunes. Este tipo de organización de la clase, incrementa la riqueza y la diversidad de las interacciones que se dan entre el alumnado y estas personas adultas.

Los grupos interactivos favorecen, al mismo tiempo, la rotación dinámica de diversas actividades de aprendizaje instrumental en una sola sesión, de manera que todo el alumnado (no solo el más aventajado) acaba realizando todas las actividades programadas para esa sesión. La riqueza de interacciones que se consiguen en el aula, por sus características, potencian la solidaridad entre el alumnado, la autoestima y las altas expectativas, y esto repercute, como se verá, en la mejora de los resultados académicos de todos y todas (INCLUD-ED Consortium, 2009). Cuando los niños y las niñas tienen la oportunidad de aprender en grupos pequeños con la presencia de diferentes personas adultas que les ayudan, incluso cuando estas personas no son profesionales de la educación, se promueve el aprendizaje. Esto se debe a que en los grupos interactivos se aprende de manera dialógica, es decir, potenciando las interacciones dialógicas basadas en un diálogo igualitario entre los niños y las niñas y las personas adultas. En los grupos interactivos este diálogo se basa en la sinceridad y en la validez de las argumentaciones que se aportan en el proceso de aprendizaje. En esas interacciones, las aportaciones del alumnado, profesorado y voluntariado con o sin estudios se valoran en función de su contenido y de cómo contribuyen al acuerdo que se consigue en el acto comunicativo: que todos los niños y niñas aprendan (Flecha et al., 2009).

\section{Actos comunicativos de poder, fracaso escolar y problemas de convivencia en la escuela}

En un instituto, se ha organizado un grupo de diversificación curricular con alumnos/as de cuarto curso de la Educación Secundaria Obligatoria (ESO) (de unos 15 y 16 años de edad). 
Son alumnos que han tenido una trayectoria en el centro de absentismo escolar, problemas de convivencia y dificultades de aprendizaje. La solución ofrecida por el profesorado es crear este grupo en el que se ofrecen unos contenidos más básicos para el alumnado. Las familias son informadas y se les explica que es mejor para sus hijos e hijas participar de este grupo para conseguir el graduado de la ESO. Sin embargo, el absentismo no se soluciona, el nivel de aprendizaje es muy bajo y poco motivador para el alumnado, los conflictos no disminuyen sino que aumentan.

La comunidad científica internacional hace décadas que ha señalado los efectos negativos de las prácticas de agrupación del alumnado y adaptaciones curriculares según su rendimiento o nivel educativo ${ }^{1}$, que pretendían corregir deficiencias del aula tradicional (Flecha, 1990; Slavin, 1991). Posteriormente, estadísticas internacionales como PISA (Programa Internacional para la Evaluación de Estudiantes) han corroborado aportaciones de la literatura científica que señalaban que se consiguen mejores resultados académicos en los centros donde no se implementa la agrupación por niveles (OECD, Organization for Economic Co-operation and Development, 2007).

Tanto en las prácticas educativas de agrupación de alumnado según su rendimiento como en aquellas que son inclusoras, la forma en cómo se da el proceso comunicativo tiene efectos en el aprendizaje. Y es en este aspecto en el que inciden los resultados del INCLUD-ED que aportamos en este artículo. Para ello, el concepto de acto comunicativo y la diferenciación entre actos comunicativos de poder y actos comunicativos dialógicos es clave, ya que permite ir más allá de lo que nos aportan los actos de habla estudiados por Austin, Searle y Habermas. Los actos comunicativos tienen en cuenta no solo lo que decimos y expresamos con palabras (en este caso la propuesta de un tipo de agrupación u otra), sino también el lenguaje no verbal, los gestos, miradas, posturas,... así como la influencia del contexto y de la estructura social, por el impacto de las jerarquías sociales que existen en los actos comunicativos dialógicos o de poder.

Esta conceptualización permite explicar mejor, por ejemplo, la exclusión que sufren los y las alumnas pertenecientes a grupos vulnerables en las escuelas donde hay prácticas segregadoras, ya que es a partir de ella que se puede analizar con mayor profundidad el tipo de interacciones que el profesorado y el resto de agentes de la comunidad educativa tienen con el alumnado desfavorecido. Desde esta conceptualización es posible además analizar las actuaciones educativas no solo desde la perspectiva de lo que se dice que estas actuaciones educativas pretenden, sino de los efectos que tienen en realidad. Los alumnos y alumnas de 15 y 16 años que en el ejemplo anterior son relegados/as a un grupo de menor nivel, sufren la estigmatización de su grupo de iguales y del profesorado, con las consecuentes repercusiones que ello conlleva en sus procesos de aprendizaje. 
Cuando alumnos/as y familiares preguntan por qué se hace esa distinción entre grupos con un nivel académico superior y grupos con un nivel académico más bajo, en muchas ocasiones reciben respuestas no sinceras ni basadas en las actuaciones de éxito avaladas por la comunidad científica internacional. No hay sinceridad en la presentación de las argumentaciones que justifican estas actuaciones en los centros educativos. No se exponen con claridad los resultados científicos aportados por investigaciones previas que demuestran que la segregación no mejora, sino que empeora, los resultados académicos del alumnado más desaventajado, siendo el alumnado perteneciente a grupos vulnerables el más perjudicado por esta práctica (INCLUDED Consortium, 2009). Esto es uno de los motivos por los que estas respuestas se convierten en actos comunicativos de poder, porque se basan en una relación de poder ejercida por parte del profesorado -quien se supone sabe cómo hay que educar al alumnado- respecto a las familias y al propio alumnado, imponiendo su decisión sobre la actuación educativa a aplicar.

En esta actuación tampoco hay consenso libre de coacciones, puesto que el alumnado no es libre de decidir a qué grupo de nivel quiere ir ni las familias se sienten libres ni con toda la información para cuestionar las decisiones del profesorado. Existe una jerarquía implícita, del marco institucional en el que se da la decisión, en la que se supone que quien sabe es solo el profesorado y son, aunque no nos gusten sus decisiones, quienes harán lo más conveniente. Cuando el cuerpo de técnicos de la educación que trabajan en el departamento de educación en la administración pública no priorizan el bienestar y el mejor aprendizaje para todos los niños y niñas, se favorece la creación de contextos en los que se encubren los elementos que favorecen el fracaso escolar. Al no estar basada en argumentos científicos ni en pretensiones de validez (Habermas, 1984, 1987), la justificación de estas actuaciones educativas se basa en especulaciones y en falsedades que se transmiten en los actos de habla y en las interacciones entre los agentes de la comunidad educativa.

Una de las personas colaboradoras de la investigación INCLUD-ED nos relata el caso de una profesora en un centro que organiza a los grupos por nivel académico. A pesar de implementar esta actuación en su clase, esta profesora defiende que no debería ser así, sino que debería organizarse el aula en base a grupos heterogéneos e incluir todos los recursos humanos posibles en el aula ordinaria, y hacer prácticas inclusoras como grupos interactivos u otras, como recomienda la comunidad científica internacional. Pero el contexto institucional en el que se encuentra, le impide aplicar las medidas educativas consideradas más exitosas. Cuando un grupo de alumnos ubicados en el grupo de bajo nivel académico le pregunta por qué están organizados así los grupos, ella les puede dar dos respuestas: una basada en actos comunicativos de poder (sin dar a conocer a los niños y niñas la verdad sobre el tema) u otra basada en actos comunicativos dialógicos. En ambos diálogos, habrá interacciones tanto dialógicas como de poder. 
Si la profesora en cuestión conoce cuáles son las actuaciones educativas de éxito avaladas por la comunidad científica internacional, podrá explicar a su alumnado que lo mejor sería que todos los grupos fueran heterogéneos y que entrasen más personas adultas en el aula o bien que hicieran desdoblamientos. Esta respuesta se daría en el marco de un acto comunicativo dialógico, basado en la sinceridad entre la profesora y los niños y niñas. Pero en este acto comunicativo dialógico también se darían interacciones de poder, puesto que aunque se esté hablando de este tema de manera sincera, los niños y niñas seguirán separados por niveles por la influencia del contexto, ya que modificar esta práctica depende de un cambio organizativo del centro que no es responsabilidad, solo, de esta profesora. La interacción de poder se da también, porque en definitiva, los niños y las niñas no deciden a qué grupo ir, puesto que esta es una decisión principalmente de la profesora, que ejerce su relación de poder sobre el alumnado.

También se puede dar el caso en el que la profesora actúe en base a un acto comunicativo de poder. Es decir, que oculte o no acabe de dar toda la información a los niños y niñas sobre la falta de validez científica de las actuaciones segregadoras. En este caso, no solo estarían presentes sino que dominarían las interacciones de poder en este acto comunicativo, puesto que no hay sinceridad en la explicación de la profesora, ni hay consenso libre de coacciones, puesto que no existe la posibilidad por parte del alumnado de llegar a un acuerdo con la profesora, ni de cuestionar la forma organizativa del aula o sus decisiones. Es decir, la decisión sobre a qué grupo debe ir cada uno de los alumnos y alumnas no es fruto de un consenso libre, sino de una imposición.

Sin embargo, en este mismo acto comunicativo de poder puede darse alguna interacción dialógica. La profesora, a la vez que no da toda la información a los niños y niñas, puede animarles a estudiar, a ayudarse, a aprender conjuntamente para conseguir el título de graduado y poder seguir estudiando después del instituto. Esta profesora puede tener interacciones dialógicas con los niños y niñas cuando sus expectativas respecto de ellos y ellas son altas, cuando es sincera al expresar su convencimiento de que todos y todas sus alumnas pueden aprender mucho, conseguir titulaciones y un futuro digno y deseado. Esta predisposición de la profesora en la interacción que tiene con su alumnado repercute en su motivación y ganas de aprender.

La influencia de la estructura social está presente, en definitiva, en todas las relaciones que se dan en el contexto escolar. Otro de los testimonios de una maestra de primaria relata como en una escuela de primaria se vieron un día en la necesidad de organizar la salida de los niños y niñas de forma excepcional. Ese día hubo una nevada que colapsó las vías de circulación de todas las ciudades y pueblos de la zona. Los familiares acudían al centro educativo a recoger a los niños y niñas de forma desordenada, en cualquier momento, para ir para casa y refugiarse del mal tiempo. El conserje de la escuela estaba intentando organizar la salida del alumnado en medio del caos, yendo a buscar a los niños y a las niñas a sus clases cada vez que llegaba un fa- 
miliar. En más de una ocasión le sugirió al director del centro la posibilidad de que los familiares pudieran acceder a las aulas a recoger ellos mismos a sus niños y niñas, sin recibir una respuesta receptiva por parte del mismo. Finalmente, una de las profesoras de una de estas aulas sugirió al director lo mismo, sin saber que el conserje ya había hecho esta propuesta. El director accedió de inmediato y permitió a los familiares ir ellos mismos a buscar a los niños y niñas a las clases. En la comunicación entre el conserje y el director y entre el director y la maestra estaba presente el esquema jerárquico de roles de la estructura social en la que nos encontramos, que hace que la opinión de la profesora pese más que la del conserje ante el director.

Los actos comunicativos de poder se dan también cuando el profesorado tiene bajas expectativas respecto de los niños y niñas y lo transmite con sus palabras, pero también con sus gestos, con sus miradas, con sus formas de tratar y de dirigirse al alumnado. Las bajas expectativas empeoran y obstaculizan el clima de aprendizaje, la convivencia y la participación. Los actos comunicativos de poder que se reflejan en estas bajas expectativas tienen lugar, por ejemplo, en los centros educativos como consecuencia del streaming, afectando a la cotidianeidad de las escuelas, a las clases, a las conversaciones entre los propios alumnos, entre alumnos y profesorado y entre este y las familias. Los niños y las niñas de los grupos de nivel académico alto se sienten superiores a los demás y viceversa. Esto repercute en las conversaciones entre ellos y ellas en los diferentes grupos: 'tú eres del grupo de los tontos', 'vosotros no haréis bachillerato', 'yo iré a la Universidad y tú no', 'en tu grupo sois muy escandalosos y os portáis peor', etc. Se genera insolidaridad entre el alumnado y como consecuencia se incrementan los problemas de convivencia y la conflictividad en las aulas, cuyas causas acaban atribuyéndose al propio alumnado estigmatizado.

Es el caso de una alumna que ha participado durante tercero y cuarto curso de secundaria, en un grupo de diversificación curricular y que ha asistido por las tardes a unos talleres en los que le han enseñado diversos oficios: peluquería, construcción, jardinería, etc. La alumna consigue el graduado escolar y se plantea seguir estudiando bachillerato. La mayoría de los profesores le cuestionan su deseo, manifestando una preocupación por ella, diciendo que creen que no podrá hacerlo y que por eso había estado dos años en un curso adaptado. Muchos le recomiendan que realice un curso formativo de grado medio, dirigido a una especialización laboral.

Con este ejemplo y los anteriores, observamos cómo los actos comunicativos de poder en las escuelas tienen importantes efectos negativos en los resultados académicos de las niñas y niños, y que promueven valores contrarios a la solidaridad y a la igualdad. Tienen serias repercusiones en la autoestima del alumnado afectado y en sus posibilidades de seguir con éxito un itinerario formativo orientado a la continuidad de los estudios académicos hasta la universidad si así se desea. 
El alumnado relegado a los grupos de menor nivel sobre los que se tienen bajas expectativas recibe a menudo los consejos del profesorado de no optar por vías formativas orientadas a la finalización de la carrera académica en la universidad. Por el contrario, se crean interacciones desalentadoras en las que son animados y animadas a decantarse por vía formativas profesionalizadoras de bajo nivel o por la inserción laboral directa al finalizar la escolarización obligatoria.

El tipo de interacción entre alumnado-profesorado es, pues, muy importante puesto que la orientación académica y profesional que se da a los y las adolescentes de secundaria es básica e influye decisivamente en la opción académica o laboral que escogen. El profesorado que ofrece esta orientación y que hace el seguimiento de los y las alumnas transmite, mediante la interacción con ellos y ellas, sus expectativas respecto a sus potencialidades académicas, ya sea de forma directa o indirecta, con el consecuente impacto sobre las decisiones que el alumnado toma.

El tipo de comunicación, articulada a través de actos comunicativos dialógicos o de poder, anima (o desanima) al alumnado, desde el inicio de su escolarización, a conseguir titulaciones y a estudiar algo que les guste y les motive. Desde el primer curso de secundaria hasta el último, si el grupo de alumnos/as es motivado por el profesorado, por su tutor/a, por la comunidad educativa para conseguir superar la secundaria, será mucho más fácil que sea así. Dedicar conversaciones basadas en actos comunicativos dialógicos, potenciadores de la libertad de los niños y niñas, donde se les escucha creando un clima de igualdad y de respeto entre ellos y ellas, donde se les anima a soñar qué desean ser cuando sean mayores, y donde se crean altas expectativas, puede hacer que un niño o niña que está fracasando en la escuela o en el instituto recupere o sienta por primera vez las ganas de estudiar junto a sus compañeros y compañeras para conseguir su sueño: ser médico, abogada, veterinaria, enfermero, etc.

Así pues, el análisis de los actos comunicativos en los centros educativos puede ayudar a comprender determinadas problemáticas escolares, como los malos resultados de grupos específicos de alumnado y los problemas de convivencia, así como emprender actuaciones de éxito para superarlos surgidas de los actos comunicativos dialógicos.

\section{Actos comunicativos dialógicos en grupos interactivos, éxito educativo, mejora de la convivencia y de la cohesión social}

Los actos comunicativos dialógicos en el contexto escolar son esenciales para transformar las dificultades en posibilidades (Freire, 1997). Según cómo se les hable y cómo se interactúe con ellos, los niños y niñas consiguen superar dificultades de aprendizaje para aprender cada día 
más y mejor, para ser más solidarios y solidarias, para convivir mejor. En definitiva, para tener más posibilidades de éxito en el mundo académico y en la vida personal.

Cuando en las actuaciones educativas dominan los actos comunicativos dialógicos se consiguen mejores resultados académicos. El proyecto INCLUD-ED nos da evidencias de ello a través del análisis de los actos comunicativos y las interacciones en los grupos interactivos, una de estas actuaciones de éxito. La inclusión de personas voluntarias en las aulas ayudando al profesor y dando apoyo a los grupos heterogéneos de alumnado es clave en esta actuación, en la que se generan interacciones dialógicas, basadas en el diálogo igualitario, donde hay sinceridad y consenso sin coacciones. Sinceridad porque, entre otras cosas, estas personas adultas que interactúan con los niños y niñas creen realmente en las potencialidades de aprendizaje de todos ellos y ellas. El clima que se crea en el aula es de altas expectativas. También hay sinceridad en estas interacciones dialógicas porque la implementación de los grupos interactivos se basa en evidencias científicas que corroboran su éxito.

Consenso sin coacciones porque, entre otras cosas, en las actividades de aprendizaje se refuerza que los niños y niñas aprendan a partir de las argumentaciones que se les dan desde un plano de igualdad, no imponiendo, sino razonando, y porque al mismo tiempo, se les anima a hacer lo mismo con sus compañeros y compañeras. De esta forma, en cada actividad de aprendizaje se va acordando entre todos y todas, y sin imposiciones, la forma de resolver los ejercicios que se están planteando en cada momento de manera que todo el alumnado pueda realizarlos y aprender con ellos.

Así pues, en los grupos interactivos se dan actos comunicativos dialógicos en los que predominan interacciones dialógicas. De este modo, los niños y niñas interiorizan que sí pueden aprender, que también pueden ayudar a los compañeros y recibir su ayuda. Esta imagen de sí mismos potenciada por los actos comunicativos dialógicos hace que niños y niñas que hasta el momento no se veían capaces de aprender, de sacar buenas notas, lo empiecen a ver posible también para ellos y ellas.

En una clase de matemáticas de una escuela de primaria, por ejemplo, se trabaja con grupos interactivos. Uno de los grupos está formado por cinco niños de 8 años y una voluntaria, ex alumna de la escuela y actual estudiante de Bachillerato en el instituto del barrio. La voluntaria tiene 16 años y resulta un referente positivo muy cercano a los niños y niñas de tercero de primaria. En este grupo heterogéneo tienen que leer y comprender un problema matemático, identificar los datos, la operación a realizar y llevarla a cabo para obtener el resultado. La voluntaria de 16 años motiva a todos los niños a resolver el problema. Primero, un niño lee, otro dice los datos, otro qué operación pueden realizar... Deciden que deben realizar una resta 
y empiezan a resolverla. Una de las niñas no ha participado hasta el momento. Los niños y las niñas de la clase no la incluyen demasiado ya que falta muchos días a la escuela y se burlan de ella. La voluntaria, que está pendiente de la niña y de las interacciones en el grupo, se da cuenta y anima a que sea ella la que explique cómo ha hecho la resta a los demás para ver si todos la tienen bien. La felicita por haberla realizado correctamente y le pide que lea ella el siguiente problema, que seguro que también lo hace muy bien. Esta interacción dialógica, bañada de las altas expectativas de esa voluntaria hacia los cinco niños del grupo interactivo, llena de unos gestos, miradas y entonaciones positivas, consigue que la niña participe como una más en el grupo, que ayude a los y las demás y que el resto le ayuden a ella.

Pero en los actos comunicativos dialógicos también se pueden dar algunas interacciones de poder. Un grupo de cinco alumnos, por ejemplo, está trabajando el tema de la diversidad lingüística. Cada alumno dice qué lenguas sabe hablar. Un alumno de Rusia dice que sabe hablar ruso y otro niño del grupo se ríe de él y le dice que entonces para qué ha venido aquí. Esta interacción es de poder y acontece en el seno de una relación de poder ejercida por el niño que se ríe sobre el niño que habla ruso. La voluntaria, una madre del barrio, interviene inmediatamente diciendo que es mucho mejor saber más lenguas que menos y que todas las personas pueden vivir en el lugar que quieran. Esta interacción dialógica hace cambiar al niño que se ríe de su compañero. Acaban hablando de las lenguas que saben hablar cada uno/a y de cuáles otras lenguas les gustaría conocer y aprender. La participación de esta madre ha hecho posible que se dé este acto comunicativo dialógico.

A través de los grupos interactivos se fomenta un uso del lenguaje no exclusor y se potencia la solidaridad entre los niños y niñas, no hablando de ella, sino haciendo que el alumnado aprenda a ser solidario a través de sus gestos, palabras y actos cuando interactúan. Es, precisamente, a través de este tipo de lenguaje, de fomentar este tipo de interacciones dialógicas, que se logra el cambio en la práctica educativa.

Esta forma de interacción también resulta beneficiosa para la superación de los prejuicios en relación al alumnado de origen inmigrante y para la inclusión del alumnado con necesidades educativas especiales. En el primer caso, la participación de voluntariado en las aulas de diferentes culturas y la interacción cercana y dialógica del mismo con los y las estudiantes contribuye a superar el desconocimiento respecto al que es diferente y consecuentemente a superar estereotipos.

En el segundo caso, la inclusión de varias personas de la comunidad interactuando dialógicamente con el alumnado juntamente con el profesor o profesora en el aula incrementa sustancialmente las posibilidades de atender las necesidades específicas de los niños y niñas y de 
incrementar el aprendizaje instrumental para todos y todas ellas, no únicamente para los y las más aventajadas.

En una clase de lengua castellana el maestro cuenta con tres personas voluntarias para realizar cuatro grupos interactivos. En uno de estos grupos participa un niño con dificultades de aprendizaje, debido a un retraso madurativo que hace que le sea más difícil comprender textos escritos y realizar actividades de comprensión lectora, entre otras tareas. En el grupo interactivo, una de las actividades consiste en leer una noticia y responder unas preguntas. Primero leen en silencio, después cada uno lee un párrafo y dialogan para encontrar la respuesta correcta a las preguntas de la actividad. La persona adulta voluntaria ya ha explicado previamente al grupo que todas y todos pueden aportar sus interpretaciones sobre lo que han leído. Saben que para aprender unos de otros tienen que escucharse con atención, y que todas las emisiones lingüísticas que se produzcan en el diálogo, les ayudarán a elaborar la respuesta correcta a la actividad. Cuando le toca el turno al niño con dificultades de aprendizaje de explicar lo que ha entendido dice "Es que no estoy seguro de haberlo entendido bien", en ese momento la compañera de su lado le dice "Seguro que algo has entendido, venga dínoslo, no tengas vergüenza!". El niño se queda en silencio. El resto de compañeros no se ríen ni se burlan de él, saben que en el grupo se respetan los turnos de palabra y se ayuda a las y los compañeros. Después de un minuto, la persona voluntaria le dice "Ya sabes que podemos encontrar la respuesta entre todas y todos, para eso necesitamos también tu idea". A continuación añade, "Si no lo has entendido bien, no pasa nada, nos sirve a todo el grupo para hablarlo y entenderlo todos mejor". Así, después de otro silencio en el niño con dificultades de aprendizaje explica su interpretación de la lectura. El grupo le mira y escucha con atención. Este acto comunicativo ha favorecido al desarrollo del aprendizaje y solidaridad en esta escuela. El niño con dificultades de aprendizaje se ha sentido escuchado, animado, fortalecido. La persona adulta voluntaria ha favorecido la promoción de actos comunicativos e interacciones dialógicas en el grupo.

En definitiva, los grupos interactivos son actos comunicativos dialógicos en los que se dan más interacciones dialógicas que de poder, y ello repercute en una mejora de los resultados académicos. Cuantas más interacciones dialógicas existan en estos actos comunicativos dialógicos, entre los niños y niñas y los diferentes miembros de la comunidad educativa, más aumentará el éxito escolar de todos y todas.

Lo mismo ocurre en cuanto a la mejora de la convivencia en el aula, en la escuela, y en el seno de las familias. Cuantas más interacciones dialógicas se den en estos contextos, mejor será la convivencia. La presencia de personas adultas que ayudan a los niños y las niñas en los grupos interactivos hace que la concentración del alumnado a la hora de realizar las actividades au- 
mente y también su motivación. Esto repercute en la reducción de la conflictividad y de las discusiones que se dan en las aulas y en el resto de espacios educativos.

En un grupo interactivo, por ejemplo, cinco niños y niñas de 12 años, en el primer curso de secundaria, están realizando una presentación en el ordenador para hacer una exposición sobre el tema de la diversidad lingüística. Cada alumno tiene su ordenador portátil. A la hora de repartirse el trabajo, no se ponen de acuerdo. Hay dos niños que quieren que se utilice su ordenador para hacer el PowerPoint, no se reparten las tareas y se pelean. La persona voluntaria que está con ellos les propone que se repartan las tareas y después unan lo que hayan hecho en un ordenador. Uno de los niños se pone muy nervioso y se enfada, se levanta del grupo y se aparta con ganas de llorar y mucha tensión física. La voluntaria se acerca y habla con él tranquilamente, le dice que no se preocupe, que es normal que se haya puesto nervioso, le pregunta si quiere quedarse un momento solo para relajarse y volver al grupo, etc. Mientras, se acerca al resto del grupo y habla con ellos sobre lo importante que es que todos estén a gusto trabajando. Los niños le dicen que cuando vuelva Karim le dirán que todo el PowerPoint se puede hacer en su ordenador, y ya le están apuntando cosas sobre el trabajo para que cuando vuelva se ponga contento.

Cuando Karim vuelve le acogen con tranquilidad y ganas y se ponen todos de acuerdo, con la ayuda de la voluntaria, sobre qué poner en cada diapositiva de la presentación. El resultado es un trabajo muy bonito sobre la diversidad lingüística en el instituto. Los y las voluntarias y la maestra del grupo les felicitan por el trabajo realizado. La participación de Karim en este grupo y las interacciones dialógicas que ha vivido con la voluntaria y el resto de compañeros y compañeras han sido muy importantes para su futuro académico. Tiene una trayectoria de un alto índice de absentismo, pero cada día tiene más ganas de venir al instituto. También ha estado en estas interacciones Jose que ha podido aportar al grupo la lengua caló porque su familia es gitana. Karim además ha aportado, palabras traducidas del castellano al árabe.

Con esta riqueza de interacciones, favorecida por la presencia de diversas personas en las aulas, y a través del diálogo igualitario que se construye en las interacciones dialógicas que predominan en los grupos interactivos, todos los niños y las niñas tienen más ganas de aprender ayudándose unos a los otros y sus resultados son mejores. Así mismo, en estas interacciones dialógicas que se fomentan en las aulas se aprende también a dialogar sobre las normas de convivencia y a consensuarlas en comunidad. Estos procesos repercuten en la promoción del aprendizaje de los valores democráticos, puesto que se aprende a compartir opiniones, a escuchar las de los y las demás y a vivir con personas diferentes. 


\section{CONCLUSIONES}

A partir de las argumentaciones presentadas en este artículo en relación a la presencia de actos comunicativos de poder y actos comunicativos dialógicos en diferentes prácticas educativas y su relación con los resultados académicos del alumnado, llegamos a dos conclusiones generales. Estas conclusiones tienen en cuenta que tanto en los actos comunicativos de poder como en los actos comunicativos dialógicos que identificamos en las actuaciones educativas se dan interacciones dialógicas e interacciones de poder, pero que existen más interacciones dialógicas en los actos comunicativos dialógicos y viceversa (más interacciones de poder en los actos comunicativos de poder), y esto tiene diferente impacto en los resultados educativos del alumnado y en la convivencia en el centro educativo.

La primera conclusión es que hay actuaciones educativas tradicionales que no tienen en cuenta la diversidad del alumnado y actuaciones educativas basadas en formas de segregación, adaptaciones curriculares o agrupación por niveles o habilidades de los niños y niñas que se basan, todas ellas, en actos comunicativos de poder. Esto quiere decir que en estas actuaciones educativas el uso del lenguaje y de la comunicación verbal y no verbal se caracteriza por una falta de sinceridad en las locuciones y una falta de consenso, o bien, en la consecución de un consenso con coacciones entre los agentes sociales implicados. Efectivamente, los niños y las niñas y sus familias desconocen en la mayoría de los casos la falta de base científica de estas actuaciones y no se encuentran en la posición de poder llegar a cuestionar la decisión de que en una escuela se hagan agrupaciones por niveles $u$ otras actuaciones segregadoras, ni pueden intervenir en la decisión de qué niños y niñas van a un grupo de nivel y cuáles van a otro.

Un elemento que contribuye a explicar estos actos comunicativos de poder es la influencia del contexto, de la estructura social, en el que hay relaciones de poder implícitas entre el profesorado y el alumnado y entre el profesorado y las familias que explican la imposición de las decisiones relativas a las actuaciones educativas que hay que aplicar en una escuela. Cuando predominan los actos comunicativos de poder, en los que se dan principalmente interacciones de poder, se genera insolidaridad entre el alumnado, falta de entendimiento entre el profesorado y las familias, y como consecuencia, se incrementan los problemas de convivencia y la conflictividad en las aulas. Se generan desconfianzas en las relaciones entre las personas que interactúan en el contexto escolar, predominan las bajas expectativas respecto las capacidades de aprendizaje del alumnado, y no se asegura que las actuaciones que se terminan implementando sean las mejores en base a argumentos, impactando todo ello en el empeoramiento de los resultados y en la perpetuación de la situación de fracaso escolar que sufren los niños y las niñas, principalmente quienes pertenecen a grupos vulnerables. 
La segunda conclusión general supone una alternativa a la situación anterior. A partir del análisis realizado, los grupos interactivos han mostrado ser una actuación educativa de éxito en la que predominan los actos comunicativos dialógicos. Aunque esta práctica no está exenta de interacciones de poder, ya que está influida por el mismo contexto y estructura social en que subyacen relaciones de poder, en ella predominan las interacciones dialógicas. Estas interacciones están basadas en la sinceridad, en la libertad y en la consecución de consensos sin coacciones. Los actos comunicativos dialógicos tienen lugar cuando los agentes sociales que intervienen en el aula (profesorado, voluntariado) adoptan una actitud activa en la generación del máximo de este tipo de interacciones dialógicas en las relaciones entre el alumnado y entre el alumnado y las personas adultas que se relacionan con los niños y las niñas.

Las escuelas que deciden implementar los grupos interactivos en sus aulas lo hacen después de conocer que esta es una práctica que está ya generando éxito educativo en otras escuelas, y se lleva a cabo como resultado del acuerdo entre diferentes colectivos de la comunidad. Por tanto, se da la condición de sinceridad, puesto que la implementación de los grupos interactivos se fundamenta en evidencias científicas, y se aplica como resultado de un consenso sin coacciones.

En la situación comunicativa que se genera en los grupos interactivos, los niños y las niñas y las personas adultas que les ayudan se sienten libres de hacer sus aportaciones y saben que estas se van a tener en cuenta al momento de decidir cuál es la mejor forma de resolver una tarea. Esto favorece la creación de altas expectativas tanto entre el mismo alumnado como entre las personas adultas, así como el surgimiento de un clima de aprendizaje que anima a todos los niños y niñas a aprender más y a superarse. En los grupos interactivos existe una actitud atenta de las personas adultas en el aula dirigida expresamente a evitar el surgimiento de interacciones de poder y a promover la riqueza de las interacciones dialógicas, lo que consigue potenciar la solidaridad y la autoestima de todos y todas, los mejores argumentos y, en consecuencia, una mejora en los resultados académicos.

\section{REFERENCIAS BIBLIOGRÁFICAS}

Aubert, A., Flecha, A., García, C., Flecha, R. \& Racionero, S. (2008). Aprendizaje dialógico en la sociedad de la información. Barcelona: Hipatia

Aubert, A., Garcia, C. \& Racionero, S. (2009). El aprendizaje dialógico. Cultura y Educación, 21(2), 128-140.

Austin, J. (1971). Cómo hacer cosas con palabras. Buenos Aires: Paidós. 
European Commission. (2006). Commission staff working document. Accompanying document to the communication from the commission to the council and to the European parliament. Efficiency and equity in European education and training systems SEC(2006)1096. Brussels: European Commission.

Flecha, R. (1990). La nueva desigualdad cultural. Barcelona: El Roure.

Flecha, R. (2009). Cambio, inclusión y calidad en las comunidades de aprendizaje. Cultura \& Educación, 21(2), 157-169.

Flecha, A., García, R., Gómez, J. \& Latorre, A. (2009). Participación en las escuelas de éxito: Una investigación comunicativa del proyecto INCLUD-ED. Cultura \& Educación, 21(2), 183-196.

Freire, P. (1997). A la sombra de este árbol. Barcelona: El Roure.

Gatt, S., Ojala, M. \& Soler, M. (en proceso). Promoting social inclusion counting with everyone: Learning communities and INCLUD-ED. International Studies in Sociology of Education.

Habermas, J. (1984). The theory of communicative action V.1. Reason and the rationalization of society. Boston: Beacon Press.

Habermas, J. (1987). The theory of communicative action. V.2. Lifeworld and system: A critique of functionalist reason. Boston: Beacon Press.

INCLUD-ED Consortium. (2009). Actions for success in schools in Europe. Brussels: European Commission.

OECD (Organization for Economic Co-operation and Development). (2007). Science competencies for tomorrow's world: Results from PISA 2006 [en línea]. Disponible en: http:// www.pisa.oecd.org/dataoecd/30/17/39703267.pdf

Racionero, S. \& Valls, R. (2007). Dialogic learning: A communicative approach to teaching and learning. En J. Kincheloe \& R. Horn (Eds.), The praeger handbook of education and psychology (pp. 548-557). Wesport, Connecticut: Greenwood Publishers.

Searle, J. (2001). Mente, lenguaje y sociedad. La filosofía del mundo real. Madrid: Alianza.

Slavin, R. (1991). Synthesis of research of cooperative learning. Educational Leadership, 48(5), 71-82.

\section{NOTAS}

1 Estas prácticas han recibido nombres diferentes como ability grouping, setting o streaming. La Comisión Europea define el streaming como la práctica de adaptar el curriculum a diferentes grupos de alumnos basándose en sus habilidades en un mismo centro educativo (European Commission, 2006). 